\title{
CONVERGENCE OF THE ABELIAN SANDPILE
}

\author{
WESLEY PEGDEN AND CHARLES K. SMART
}

\begin{abstract}
The Abelian sandpile growth model is a diffusion process for configurations of chips placed on vertices of the integer lattice $\mathbb{Z}^{d}$, in which sites with at least $2 d$ chips topple, distributing 1 chip to each of their neighbors in the lattice, until no more topplings are possible. From an initial configuration consisting of $n$ chips placed at a single vertex, the rescaled stable configuration seems to converge to a particular fractal pattern as $n \rightarrow \infty$. However, little has been proved about the appearance of the stable configurations. We use PDE techniques to prove that the rescaled stable configurations do indeed converge to a unique limit as $n \rightarrow \infty$. We characterize the limit as the Laplacian of the solution to an elliptic obstacle problem.
\end{abstract}

\section{INTRODUCTION}

The Abelian sandpile growth model is a diffusion process for configurations of chips placed on vertices of the $d$-dimensional integer lattice $\mathbb{Z}^{d}$. When a vertex in $\mathbb{Z}^{d}$ topples, it loses $2 d$ chips itself, adding one chip to each of its $2 d$ neighbors in the lattice. For any finite nonnegative initial distribution of chips, one can topple vertices with at least $2 d$ chips until no more such topplings are possible, and, remarkably, neither the final configuration nor the number of topplings which occur at each vertex depend on the order in which topplings are performed - the process is in this sense Abelian, as first noticed in [4,5].

Introduced in [1, the sandpile growth model has been the subject of extensive study over the past two decades, both on $\mathbb{Z}^{d}$ and on general graphs. Nevertheless, many of the earliest and most fundamental questions regarding the sandpile remain unanswered (see e.g. 12]). Some of the most nagging concern the final configuration $s_{n}: \mathbb{Z}^{d} \rightarrow\{0,1, \ldots, 2 d-1\}$ obtained from starting with $n$ chips placed at the origin of the integer lattice. Terminal configurations for several values of $n$ and $d=2$ are shown in Figure 1. Rescaled by a factor of $n^{1 / d}$, the piles seem to converge to a fractal-like limiting image, but essentially no progress has been made in proving precise things about this limit. (For example, there is no proof that there are regions in the limit which have constant value 3, no proof that its boundary does not converge to a circle, etc.) In this paper, we show at least that the limit exists as an object to study - the rescaled sandpile does converge.

Theorem 1.1. The rescaled sandpiles $\bar{s}_{n}(x):=s_{n}\left(n^{1 / d} x\right)$ converge weakly-* to a function $s \in L^{\infty}\left(\mathbb{R}^{d}\right)$ as $n \rightarrow \infty$. Moreover, the limit $s$ satisfies $\int_{\mathbb{R}^{d}} s d x=1$, $0 \leq s \leq 2 d-1$, and $s=0$ in $\mathbb{R}^{d} \backslash B_{R}$ for some $R>0$.

Date: October 22, 2018.

2010 Mathematics Subject Classification. 60K35, 35R35.

Key words and phrases. abelian sandpile, asymptotic shape, obstacle problem.

The authors were partially supported by NSF grants DMS-1004696 and DMS-1004595. 

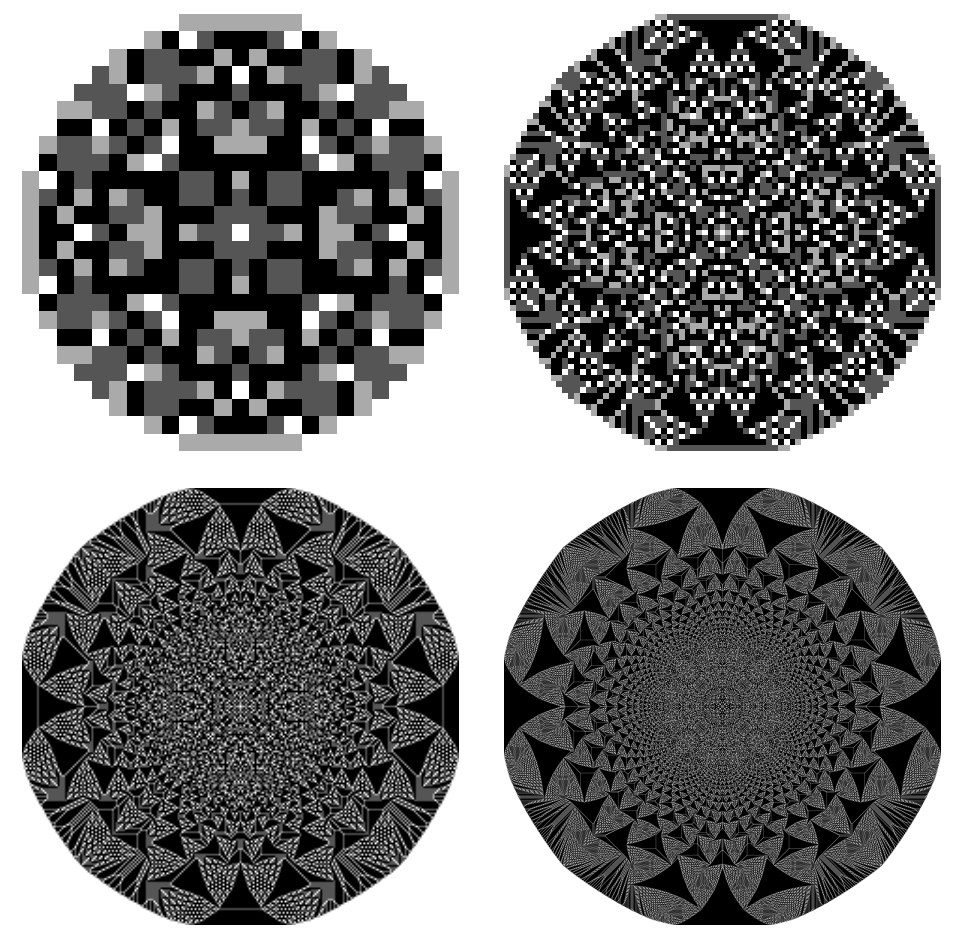

Figure 1. Sandpiles started from $n=10^{3}, n=10^{4}, n=10^{5}$, and $n=10^{6}$ chips placed at the origin of $\mathbb{Z}^{2}$, rescaled by factors of $n^{1 / 2}$. Sites with $0,1,2$, and 3 chips are represented by white, light gray, dark gray, and black, respectively.

See Remark 4.3 for the characterization the limiting $s \in L^{\infty}\left(\mathbb{R}^{d}\right)$ as the Laplacian of the solution of an elliptic obstacle problem.

We remark that weak-* convergence seems to be the correct notion of convergence for the rescaled sandpiles. Recall that $L^{\infty}\left(\mathbb{R}^{d}\right)$ denotes the space of bounded measurable functions on $\mathbb{R}^{d}$ and that $C_{0}\left(\mathbb{R}^{d}\right)$ denotes the continuous functions on $\mathbb{R}^{d}$ with compact support. A sequence of functions $\bar{s}_{n} \in L^{\infty}\left(\mathbb{R}^{d}\right)$ converges weakly-* to a function $s \in L^{\infty}\left(\mathbb{R}^{d}\right)$ in $L^{\infty}\left(\mathbb{R}^{d}\right)$ if

$$
\int_{\mathbb{R}^{d}} \bar{s}_{n} \varphi d x \rightarrow \int_{\mathbb{R}^{d}} s \varphi d x \quad \text { as } n \rightarrow \infty,
$$

for all test functions $\varphi \in C_{0}\left(\mathbb{R}^{d}\right)$. If we look closely at the gray regions of the sandpiles (see Figure 2), we see that they consist of rapidly oscillating patterns, as first noticed in 13. It seems clear that no kind of pointwise convergence of the sandpiles can hold, assuming this behavior persists as $n \rightarrow \infty$. Instead, the sandpiles converge in the sense that the oscillating regions converge to their average value; there is some limiting image $s$ in which the "colors" of points are real numbers, approximated in the sequence $\bar{s}_{n}$ by patterns of integers. The convergence of a sequence of bounded functions in local average value is precisely what weak-* convergence in $L^{\infty}\left(\mathbb{R}^{d}\right)$ is designed to capture 6 . 


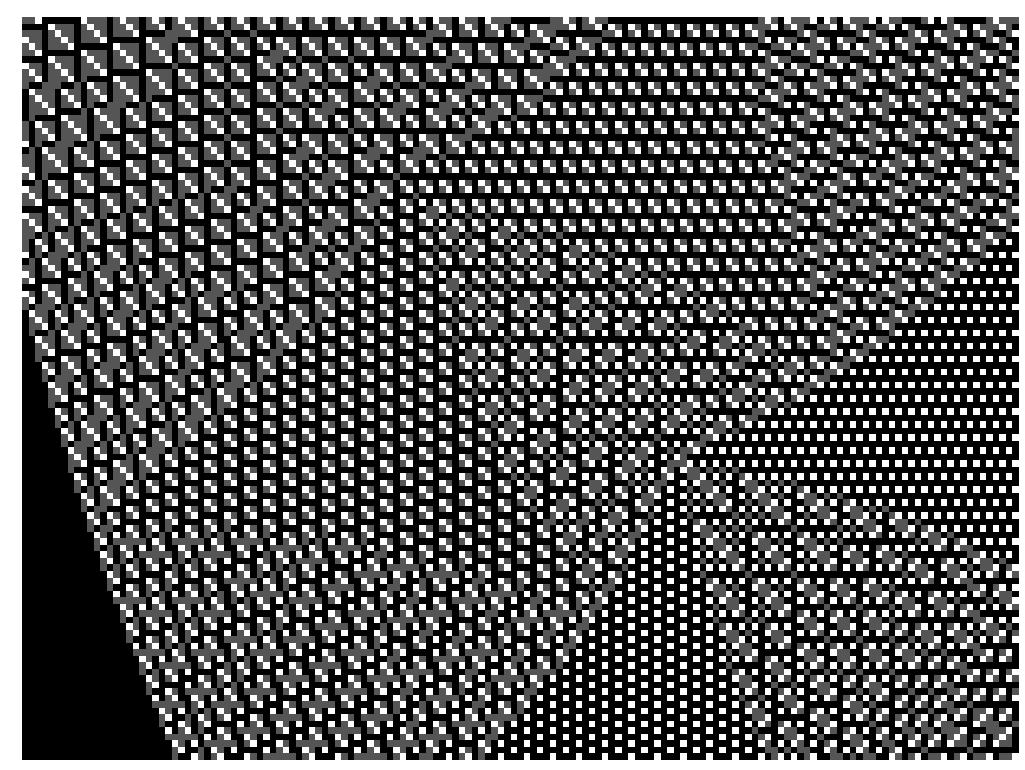

Figure 2. Periodic structure in the "gray" regions of the $n=$ $16 \cdot 10^{6}$ sandpile on $\mathbb{Z}^{2}$. Notice the patterned triangular shapes.

Note that the properties of $s$ stated in the second part of the theorem are to demonstrate that the weak-* convergence is not for trivial reasons such as a poor choice of the rescaling: the mass of the limit is 1 and is not being "rescaled away"; similarly, the rescaled sandpiles are contained within a ball of a fixed size. The best known upper bound on the diameter of the this ball [1] is that for every $\varepsilon>0$ there is a $C=C(\varepsilon, d)>0$ such that

$$
\left\{s_{n}>0\right\} \subseteq B_{\left((d-\varepsilon)\left|B_{1}\right|\right)^{-1 / d} n^{1 / d}+C}
$$

for all $n>0$. Here $\left|B_{1}\right|$ denotes the volume of the unit ball.

Although our goal will be the characterization of final chip configurations, we will really be studying the number of topples which occur at each vertex as an initial configuration stabilizes. Given an initial configuration of chips and some finite sequence $\left\{p_{i}\right\} \subset \mathbb{Z}^{d}$ of points at which we topple piles of chips, the sequence is legal if the sequence never topples any vertex with fewer than $2 d$ chips, and stabilizing if the final configuration has fewer than $2 d$ chips at each vertex. Given any legal sequence $p_{1}, \ldots, p_{s}$ and any stabilizing sequence $q_{1}, \ldots, q_{t}$ both beginning from some fixed intial configuation, we must have $p_{1}=q_{i_{1}}$ for some $i_{1}$, since there must be at least $2 d$ chips at the point $p_{1}$ in the initial configuration, since $\left\{p_{i}\right\}$ is legal, and these chips must topple in any stabilizing sequence. Since the permuted sequence $q_{i_{1}}, q_{1}, \ldots, q_{i_{1}-1}, q_{i_{1}+1}, \ldots, q_{t}$ is also stabilizing, we can apply the same argument again from the configuration obtained after toppling just at $p_{1}=q_{i_{1}}$, to get that there must be a $q_{i_{2}}=p_{2}$. Continuing in this manner, we obtain that the sequence $\left\{p_{i}\right\}$ is a permutation of some subsequence of $\left\{q_{i}\right\}$.

The above argument, a simplification of what appeared in [5], implies that for any fixed initial configuration of chips, any two legal stabilizing sequences must be 
permutations of each other. Coupled with the fact that any finite initial configuration of chips on $\mathbb{Z}^{d}$ will eventually stabilize after some legal sequence of topples, this implies that there is a well-defined odometer function $v: \mathbb{Z}^{d} \rightarrow \mathbb{N}$ associated to any initial configuration, which counts the number of topples which will occur at points of the lattice in any legal stabilizing sequence of topples. This also implies the Abelian property of the sandpile, as the final chip configuration $s$ is uniquely determined by the odometer function. Note that it can be computed simply as

$$
s(x)=\eta(x)+\sum_{y \sim x}(v(y)-v(x)),
$$

where $\eta$ is the initial configuration of chips, and the sum is taken over the $2 d$ neighbors $y$ of $x$ in the lattice $\mathbb{Z}^{d}$. Thus we have

$$
s=\eta+\Delta^{1} v,
$$

where $\Delta^{1}$ is the $(2 d+1)$-point discrete Laplacian on $\mathbb{Z}^{d}$.

The starting point of our proof is the least action principle formulated in 7 . Suppose that $u$ is the odometer function for some initial configuration $\eta$ of chips, and $v: \mathbb{Z}^{d} \rightarrow \mathbb{N}$ is any function that satisfies

$$
\eta+\Delta^{1} v \leq 2 d-1 .
$$

The least action principle states that we must have $u \leq v$. Note that this follows immediately from the fact that legal sequences are permutations of subsequences of stabilizing sequences, since $u$ corresponds to a stabilizing and legal sequence, while $v$ corresponds to a stablizing, not necessarily legal sequence.

The important consequence of the least action principle for us is that the odometer function associated with a configuration is the pointwise minimum of all functions $v: \mathbb{Z}^{d} \rightarrow \mathbb{N}$ satisfying $\eta+\Delta^{1} v \leq 2 d-1$, where $\eta: \mathbb{Z}^{d} \rightarrow \mathbb{N}$ is the initial configuration of chips. In our case, if $v_{n}$ is the odometer function resulting from an initial configuration of $n$ chips at the origin, we have

$$
v_{n}=\min \left\{v: \mathbb{Z}^{d} \rightarrow \mathbb{N} \mid n \delta_{0}+\Delta^{1} v \leq 2 d-1\right\},
$$

where $\delta_{0}$ is the characteristic function of the set $\{0\} \subset \mathbb{Z}^{d}$. The final configuration of chips is then

$$
s_{n}:=n \delta_{0}+\Delta^{1} v_{n} .
$$

This description of $v_{n}$ and $s_{n}$, together with standard estimates for the $(2 d+1)$ point Laplacian $\Delta^{1}$, will easily give convergence of the rescaled $\bar{s}_{n}$ and the rescaled odometer function $\bar{v}_{n}(x):=n^{-2 / d} v_{n}\left(n^{1 / d} x\right)$ along subsequences $n_{k} \rightarrow \infty$.

To obtain convergence, we show that the limiting $s$ and $v$ are independent of the choice of subsequence. Assuming there are two distinct limits $v$ and $v^{\prime}$, we use the regularity theory of the Laplacian to select a point $x \in \mathbb{R}^{d} \backslash\{0\}$ where $v$ and $v^{\prime}$ are sufficiently smooth and $v-v^{\prime}$ is strictly concave. We then select sufficiently close finite approximations $v \approx \bar{v}_{n}$ and $v^{\prime} \approx \bar{v}_{n^{\prime}}$ and use $\bar{v}_{n^{\prime}}$ to "lower" $\bar{v}_{n}$, contradicting the least action principle for $v_{n}$. The difficultly lies in the fact that $\bar{v}_{n}$ and $\bar{v}_{n^{\prime}}$ are defined on possibly incompatible lattices. We overcome this obstacle using an approximation argument (see Lemma 4.1) that allows us to change the scale of $\bar{v}_{n^{\prime}}$.

Acknowledgements. The authors would like to thank Robert V. Kohn for several useful discussions and Lionel Levine for drawing our attention to the problem and for making comments on an early draft. 


\section{Preliminaries}

2.1. Notation. We write $h:=n^{-1 / d}$. We define the open ball $B_{r}(x):=\left\{y \in \mathbb{R}^{d} \mid\right.$ $|y-x|<r\}$ for $r>0$ and $x \in \mathbb{R}^{d}$ and define $B_{r}:=B_{r}(0)$. We write $\partial \Omega$ for the boundary of an open set $\Omega \subseteq \mathbb{R}^{d}$. A function $u \in C\left(\mathbb{R}^{d}\right)$ is twice differentiable at a point $x \in \mathbb{R}^{d}$ if there is a vector $D u(x) \in \mathbb{R}^{d}$ and a symmetric $d \times d$ matrix $D^{2} u(x) \in S_{d}$ such that

$$
u(y)=u(x)+D u(x) \cdot(y-x)+\frac{1}{2}(y-x)^{t} D^{2} u(x)(y-x)+o\left(|y-x|^{2}\right) .
$$

We write $y \sim_{h} x$ when $y-x \in h \mathbb{Z}^{d}:=\left\{h x \mid x \in \mathbb{Z}^{d}\right\}$ and $|y-x|=h$. Note that $y \sim_{h} x$ if and only if $y=x \pm h e_{i}$ for some coordinate vector $e_{i}$. Given a subset of the lattice $E \subseteq h \mathbb{Z}^{d}$, we write

$$
\partial^{h} E:=\left\{y \in h \mathbb{Z}^{d} \backslash E \mid y \sim_{h} x \text { for some } x \in E\right\}
$$

for its lattice boundary.

2.2. Interpolation convention. Throughout this article, we consider sequences of functions $u_{n}: h \mathbb{Z}^{d} \rightarrow \mathbb{R}$. We implicitly extend all such functions to $\mathbb{R}^{d}$ via nearest-neighbor interpolation. That is, we set

$$
u_{n}(x):=u_{n}\left(h\left\lfloor h^{-1} x\right\rceil\right)
$$

to be the value of $u_{n}$ at the lattice point $h\left\lfloor h^{-1} x\right\rceil \in h \mathbb{Z}^{d}$ closest to $x \in \mathbb{R}^{d}$. Here $\lfloor\cdot\urcorner$ denotes coordinate-wise rounding to the nearest integer (rounding down for ties). This convention allows us to make sense of statements like " $u_{n} \rightarrow u \in C\left(\mathbb{R}^{d}\right)$ locally uniformly as $n \rightarrow \infty$ " when the functions $u_{n}$ are only defined on the lattice $h \mathbb{Z}^{d}$.

2.3. The Laplacian. The Laplacian

$$
\Delta u:=\operatorname{trace}\left(D^{2} u\right)=\sum_{i=1}^{d} \frac{\partial^{2}}{\partial x_{i}^{2}} u,
$$

and the $(2 d+1)$-point discrete Laplacian

$$
\Delta^{h} u(x):=\frac{1}{h^{2}} \sum_{y \sim_{h} x}(u(y)-u(x))
$$

play a central role in our analysis of the sandpile. Recall that if $\varphi \in C^{\infty}\left(\mathbb{R}^{d}\right)$, then $\Delta^{h} \varphi \rightarrow \Delta \varphi$ locally uniformly in $\mathbb{R}^{d}$ as $h \rightarrow 0$.

From [10], we know that the discrete Laplacian $\Delta^{h}$ has a "fundamental solution" $\Phi_{n}: h \mathbb{Z}^{d} \rightarrow \mathbb{R}$ that satisfies

$$
\Delta^{h} \Phi_{n}(x)= \begin{cases}-n & \text { if } x=0 \\ 0 & \text { if } x \neq 0\end{cases}
$$

for all $x \in h \mathbb{Z}^{d}$. The function $\Phi_{n}(x)$ can be realized as a normalization of the expected number of visits of a random walk to the point $x$ on the lattice $h \mathbb{Z}^{d}$, adjusting for recurrences in the case $d=2$. As $n \rightarrow \infty, \Phi_{n} \rightarrow \Phi \in C^{\infty}\left(\mathbb{R}^{d} \backslash\{0\}\right)$ locally uniformly in $\mathbb{R}^{d} \backslash\{0\}$, where

$$
\Phi(x):= \begin{cases}\left(d(d-2)\left|B_{1}\right|\right)^{-1}|x|^{2-d} & \text { if } d \geq 3, \\ -(2 \pi)^{-1} \log |x| & \text { if } d=2,\end{cases}
$$


is the "fundamental solution" of the Laplacian. We use the convergence of $\Phi_{n} \rightarrow \Phi$ to resolve the formation of a singularity in $\bar{v}_{n}$ as $n \rightarrow \infty$.

The discrete Laplacian is monotone: $\Delta^{h} u(x)$ is decreasing in $u(x)$ and increasing in $u(y)$ for any $y \sim_{h} x$. An obvious consequence of monotonicity is the following estimate for the discrete Laplacian of the point-wise minimum of two functions:

Proposition 2.1. If $u, v: h \mathbb{Z}^{d} \rightarrow \mathbb{R}, w:=\min \{u, v\}$, and $w(x)=u(x)$, then $\Delta^{h} w(x) \leq \Delta^{h} u(x)$.

A less obvious consequence of monotonicity is the fact that $\Delta^{h}$ has a maximum principle [9]:

Proposition 2.2. For $u, v: h \mathbb{Z}^{d} \rightarrow \mathbb{R}, E \subseteq h \mathbb{Z}^{d}$ finite, and $\Delta^{h} u \geq \Delta^{h} v$ in $E$, we have $\max _{\partial^{h} E}(u-v) \geq \max _{E}(u-v)$. That is, the maximum difference must occur on the lattice boundary.

Finally, we recall a consequence of the standard a priori estimates for the finite difference Laplacian and the Arzela-Ascoli theorem 9]:

Proposition 2.3. If $u_{n}: h \mathbb{Z}^{d} \rightarrow \mathbb{R}$ is a sequence of functions that satisfies

$$
\max _{B_{1}}\left|u_{n}\right| \leq C \quad \text { and } \quad \max _{B_{1}}\left|\Delta^{h} u_{n}\right| \leq C
$$

then for every sequence $n_{k} \rightarrow \infty$ there is subsequence $n_{k_{j}} \rightarrow \infty$ and a function $u \in C\left(B_{1}\right)$ such that $u_{n_{k_{j}}} \rightarrow u$ locally uniformly in $B_{1}$ as $j \rightarrow \infty$.

After we make some additional preparations, the above two propositions will allow us to show that the $\bar{v}_{n}$ converge along subsequences. It remains to recall some standard facts about the continuum Laplacian which we use to study the regularity of the possible limits of the $\bar{v}_{n}$.

If $\Omega \subseteq \mathbb{R}^{d}$ is open and $s \in L^{\infty}(\Omega)$, then we say that $u \in C(\Omega)$ is a weak solution (see [8]) of the continuum Laplace equation

$$
\Delta u=s \text { in } \Omega
$$

if and only if

$$
\int_{\Omega} u \Delta \varphi d x=\int_{\Omega} s \varphi d x
$$

for all test functions $\varphi \in C_{0}^{\infty}(\Omega)$. Note that if $u$ and $s$ happen to be smooth, then we can we can intergrate by parts to show that this is equivalent to $\Delta u=s$ holding pointwise.

The following is a consequence of a much stronger result from the theory of singular operators 3 , Chapter III Theorem 4]:

Proposition 2.4. If $s \in L^{\infty}\left(B_{1}\right)$ and $u \in C\left(B_{1}\right)$ is a weak solution of $\Delta u=s$ in $B_{1}$, then $u$ is twice differentiable and $\Delta u=s$ almost everywhere in $B_{1}$.

Having bounded Laplacian also implies that a function must be strictly concave on a set of positive measure in any neighborhood of a strict local maximum 2 , Theorem 3.2]:

Proposition 2.5. If $s \in L^{\infty}\left(B_{1}\right)$ and $u \in C\left(B_{1}\right)$ is a weak solution of $\Delta u=s$ in $B_{1}$, and $\sup _{B_{1}} u>\sup _{\partial B_{1}} u$, then the set

$$
\left\{x \in B_{1} \mid u(x)>\sup _{\partial B_{1}} u, u \text { is twice differentiable at } x \text {, and } D^{2} u(x)<0\right\},
$$

has positive measure. 


\section{Convergence Along subsequences}

Note from Section 1, and in particular (1.1), that the sandpile $s_{n}$ satisfies

$$
\int_{\mathbb{R}^{d}} s_{n} d x=n, \quad 0 \leq s_{n} \leq 2 d-1 \quad \text { and } \quad\left\{s_{n}>0\right\} \subseteq B_{h^{-1} R},
$$

for some $R>0$ independent of $n$. These facts, together with the least action principle and the results in Section 2, are enough to prove convergence of the rescaled sandpile along subsequences.

Recall that $\bar{s}_{n}(x):=s\left(h^{-1} x\right)$ and $\bar{v}_{n}(x):=h^{2} v_{n}\left(h^{-1} x\right)$, and define

$$
\bar{w}_{n}:=\bar{v}_{n}-\Phi_{n} .
$$

Since $\Delta^{h}$ is linear and $\Delta^{h} \Phi_{n}=-n \delta_{0}$, we have

$$
\Delta^{h} \bar{w}_{n}=n \delta_{0}+\Delta^{h} \bar{v}_{n}=\bar{s}_{n} \quad \text { in } \mathbb{R}^{d} .
$$

Lemma 3.1. For every sequence $n_{k} \rightarrow \infty$ there is a subsequence $n_{k_{j}} \rightarrow \infty$ and functions $w \in C\left(\mathbb{R}^{d}\right)$ and $s \in L^{\infty}\left(\mathbb{R}^{d}\right)$ such that $w$ is a weak solution of $\Delta w=s$ in $\mathbb{R}^{d}, \bar{w}_{n_{k_{j}}}$ converges locally uniformly to $w$ in $C\left(\mathbb{R}^{d}\right)$, and $\bar{s}_{n_{k_{j}}}$ converges weakly-* to $s$ in $L^{\infty}\left(\mathbb{R}^{d}\right)$.

Note that this lemma holds for any sequence $w_{n}: h \mathbb{Z}^{d} \rightarrow \mathbb{R}$ such that $w_{n}$ and $\Delta^{h} w_{n}$ are locally uniformly bounded. Uniqueness of the limit is where our analysis is particular to the sandpile.

Proof. Since $\left\{\bar{v}_{n}>0\right\} \subseteq B_{R}$ and $\bar{v}_{n} \geq 0$, we therefore have

$$
\bar{w}_{n} \geq-\Phi_{n} \quad \text { in } h \mathbb{Z}^{d} \quad \text { and } \quad \bar{w}_{n}=-\Phi_{n} \quad \text { on } h \mathbb{Z}^{d} \backslash B_{R} .
$$

If we set $E:=h \mathbb{Z}^{d} \cap B_{R}$, then the test functions

$$
\varphi(x):=|x|^{2}-(R+h)^{2}+\inf _{\partial^{h} E}-\Phi_{n} \quad \text { and } \quad \psi(x):=\sup _{\partial^{h} E}-\Phi_{n},
$$

satisfy $\varphi \leq \bar{w}_{n} \leq \psi$ on $\partial^{h} E$ and $2 d=\Delta^{h} \varphi \geq \Delta^{h} \bar{w}_{n} \geq \Delta^{h} \psi=0$ in $E$. It follows from the maximum principle (Proposition 2.2) that

$$
|x|^{2}-(R+h)^{2}+\inf _{\partial^{h} E}-\Phi_{n} \leq \bar{w}_{n}(x) \leq \sup _{\partial^{h} E}-\Phi_{n} \quad \text { for all } x \in B_{R} .
$$

For any $R^{\prime}>R$, we know that $\Phi_{n} \rightarrow \Phi$ uniformly in $B_{R^{\prime}} \backslash B_{R}$ as $n \rightarrow \infty$. Thus the sequence $\bar{w}_{n}$ satisfies

$$
\left\|\bar{w}_{n}\right\|_{L^{\infty}\left(B_{R^{\prime}}\right)} \leq C
$$

for some $C=C\left(R^{\prime}\right)>0$ and all $n>0$. Since we also have $\left|\Delta^{h} \bar{w}_{n}\right| \leq 2 d-1$, Proposition 2.3 implies the existence of a subsequence $n_{k_{j}}$ and a function $w \in C\left(\mathbb{R}^{d}\right)$ such that $\bar{w}_{n_{j}} \rightarrow w$ locally uniformly as $j \rightarrow \infty$. Since any uniformly bounded sequence of functions converges weakly-* along subsequences [6, Page 7], we may select a further subsequence and a function $s \in L^{\infty}\left(\mathbb{R}^{d}\right)$ such that $\bar{s}_{n_{j}}$ converges weakly-* to $s$ as $j \rightarrow \infty$.

For any $\varphi \in C_{0}^{\infty}\left(\mathbb{R}^{d}\right)$, we have

$$
\int_{\mathbb{R}^{d}} \varphi s_{n_{j}} d x=\int_{\mathbb{R}^{d}} \varphi \Delta^{h_{j}} \bar{w}_{n_{j}} d x=\int_{\mathbb{R}^{d}}\left(\Delta^{h_{j}} \varphi\right) \bar{w}_{n_{j}} d x,
$$

where the second equality comes from the discrete integration by parts formula, which an be carried out in this case by writing $\Delta^{h_{j}} \bar{w}_{n}$ as a finite sum that commutes with the integral. 
Since $\bar{w}_{n_{j}} \rightarrow w$ locally uniformly in $\mathbb{R}^{d}$, it converges uniformly on any closed ball, and therefore uniformly on any bounded neighborhood of the support of $\varphi$. Since we also have $\Delta^{h} \varphi \rightarrow \Delta \varphi$ uniformly in $\mathbb{R}^{d}$, we see that the right-hand side of 3.2 converges to $\int_{\mathbb{R}^{d}} w \Delta \varphi d x$ as $j \rightarrow \infty$. The left-hand side converges to $\int_{\mathbb{R}^{d}} s \varphi d x$ by the definition of weak-* convergence. Thus $w$ is a weak solution of $\Delta w=s$ in $\mathbb{R}^{d}$ by definition.

An immediate consequence of Lemma 3.1 is that $\bar{v}_{n_{j}} \rightarrow v:=w+\Phi$ locally uniformly in $\mathbb{R}^{d} \backslash\{0\}$ as $j \rightarrow \infty$. However, what we obtained is strictly stronger, since it allows us to resolve the structure of the singularity of $v$.

\section{Convergence}

As discussed in Section 1, we would like to compare two limits $\bar{v}_{n_{k}} \rightarrow v$ and $\bar{v}_{n_{k}^{\prime}} \rightarrow v^{\prime}$ along different sequences $n_{k} \rightarrow \infty$ and $n_{k}^{\prime} \rightarrow \infty$ by comparing their finite approximations, in spite of the fact that their approximations $\bar{v}_{n_{k}}$ and $\bar{v}_{n_{k}^{\prime}}$ may not be defined on the same scale. To deal with this, we could try construct an asymptotic expansion of the convergence $\bar{v}_{n_{k}} \rightarrow v$ in a neighborhood of each point $x \in \mathbb{R}^{d} \backslash\{0\}$ where $v$ is twice differentiable. That is, we could try to find a radius $r>0$ and a function $u: \mathbb{Z}^{d} \rightarrow \mathbb{Z}$ such that $\Delta^{1} u \leq 2 d-1$ and

$$
\bar{v}_{n_{k}}(y)=v(x)+D v(x) \cdot(y-x)+h_{k}^{2} u\left(h_{k}^{-1}(y-x)\right)+o\left(|y-x|^{2}\right)+o(1)
$$

for all $y \in B_{r}(x)$ and $k>0$. (Note that $o(1)$ is with respect to $k \rightarrow \infty$ while $o\left(|y-x|^{2}\right)$ is with respect to the distance $|y-x| \rightarrow 0$.) The existence of such an expansion would make it easy to approximate $v$ on different scales in $B_{r}(x)$. Indeed, while $v$ only has approximations $\bar{v}_{n_{k}}$ defined on the scales $h_{k}$, the right-hand side of 4.1 is valid for any scale $h>0$.

Unfortunately, we do not know how to prove that an asymptotic expansion of the form 4.1 exists in general. Instead we construct a one-sided expansion, which is sufficient for our purposes. Note that this lemma is applied below to $v_{n}$ that have been translated and shifted and in particular may take negative values.

Lemma 4.1. Suppose $v_{n}: \mathbb{Z}^{d} \rightarrow \mathbb{Z}$ is any sequence of functions such that $\Delta^{1} v_{n} \leq$ $K \in \mathbb{Z}$. Suppose $v \in C\left(B_{r}\left(x_{0}\right)\right)$ for some $r>0$ and the rescalings $\bar{v}_{n}(x):=$ $h^{2} v_{n}\left(h^{-1} x\right)$ converge uniformly $\bar{v}_{n_{k}} \rightarrow v$ in $B_{r}\left(x_{0}\right)$ along some sequence $n_{k} \rightarrow \infty$. If $v$ is twice differentiable at $x_{0}$, then for every $\varepsilon>0$ there is a function $u: \mathbb{Z}^{d} \rightarrow \mathbb{Z}$ such that

$$
\Delta^{1} u(x) \leq K \quad \text { and } \quad u(x) \geq \frac{1}{2} x^{t}\left(D^{2} v\left(x_{0}\right)-\varepsilon I\right) x \quad \text { for all } x \in \mathbb{Z}^{d} .
$$

Proof. Replacing $v_{n}$ if necessary by

$$
v_{n}^{\prime}(x):=v_{n}\left(x+\left\lfloor h^{-1} x_{0}\right\rceil\right)-v_{n}\left(\left\lfloor h^{-1} x_{0}\right\rceil\right)-\left\lfloor h^{-1} D v\left(x_{0}\right)\right\rceil \cdot x,
$$

we may assume that $x_{0}=0, v(0)=0, D v(0)=0$, and $v_{n}(0)=0$.

Since $v$ is twice differentiable at 0 , we can make $r>0$ smaller and select a large $n=n_{k}$ such that

$$
\sup _{B_{r}}\left|\bar{v}_{n}-\varphi\right| \leq \varepsilon r^{2}
$$

where $\varphi(x):=\frac{1}{2} x^{t} D^{2} v(0) x$. Noting that $h^{2} \varphi\left(h^{-1} x\right)=\varphi(x)$, we can undo the scaling of $\bar{v}_{n}$, obtaining

$$
\sup _{B_{h^{-1} r}}\left|v_{n}-\varphi\right| \leq \varepsilon h^{-2} r^{2}
$$


Thus, if we define $\psi(x):=\frac{1}{2} x^{t}\left(D^{2} v(0)-32 \varepsilon I\right) x$, we have $\varphi-\psi=16 \varepsilon|x|^{2}$ and therefore

$$
\begin{cases}v_{n} \leq \psi+2 \varepsilon h^{-2} r^{2} & \text { in } B_{h^{-1} r / 4} \\ v_{n} \geq \psi+3 \varepsilon h^{-2} r^{2} & \text { in } B_{h^{-1} r} \backslash B_{h^{-1} r / 2}\end{cases}
$$

We define $u$ as an overlapping pointwise minimum of translated and tilted copies of $v_{n}$,

where

$$
u(x):=\min \left\{v_{n, y}(x) \mid y \in \mathbb{Z}^{d} \cap B_{h^{-1} r}(x)\right\},
$$

$$
v_{n, y}(x):=v_{n}(x-y)+\lfloor D \psi(y)\rceil \cdot(x-y)+\lfloor\psi(y)\rceil .
$$

The inequalities 4.2 guarantee that the overlapping works out correctly. Indeed, for $x \in B_{h^{-1} r / 4}(y)$, we compute

$$
\begin{aligned}
v_{n, y}(x) & =v_{n}(x-y)+\lfloor D \psi(y)\rceil \cdot(x-y)+\lfloor\psi(y)\rceil \\
& \leq \psi(x-y)+\lfloor D \psi(y)\rceil \cdot(x-y)+\lfloor\psi(y)\rceil+2 \varepsilon h^{-2} r^{2} \\
& \leq \psi(x-y)+D \psi(y) \cdot(x-y)+\psi(y)+2 \varepsilon h^{-2} r^{2}+d^{1 / 2} h^{-1} r+1 \\
& =\psi(x)+2 \varepsilon h^{-2} r^{2}+d^{1 / 2} h^{-1} r+1 .
\end{aligned}
$$

A similar computation shows

$$
v_{n, y} \geq \psi+3 \varepsilon h^{-2} r^{2}-d^{1 / 2} h^{-1} r-1 \quad \text { in } B_{h^{-1} r}(y) \backslash B_{h^{-1} r / 2}(y) .
$$

By making $n$ larger, we may assume that $h^{-1} r / 4>1$ and

$$
2 \varepsilon h^{-2} r^{2}+d^{1 / 2} h^{-1} r+1<3 \varepsilon h^{-2} r^{2}-d^{1 / 2} h^{-1} r-1 .
$$

It follows that

$$
u(x)=\min \left\{v_{n, y}(x) \mid y \in \mathbb{Z}^{d} \cap B_{h^{-1} r / 2}(x)\right\},
$$

and therefore

$$
u=\min \left\{v_{n, y} \mid y \in \mathbb{Z}^{d} \cap B_{3 h^{-1} r / 4}(x)\right\} \quad \text { in } B_{h^{-1} r / 4}(x),
$$

for all $x \in \mathbb{Z}^{d}$. Since $\Delta^{1} v_{n, y} \leq K$ and $h^{-1} r / 4>1$, Proposition 2.1 implies that $\Delta^{1} u(x) \leq K$. Moreover, it is easy to check that $u-\psi$ is bounded from below.

We are now in a position to prove Theorem 1.1. In fact, we prove something slightly stronger.

Theorem 4.2. There are functions $w \in C\left(\mathbb{R}^{d}\right)$ and $s \in L^{\infty}\left(\mathbb{R}^{d}\right)$ such that $\bar{s}_{n}$ converges weakly-* to $s$ in $L^{\infty}\left(\mathbb{R}^{d}\right), \bar{w}_{n}$ converges locally uniformly to $w$ in $C\left(\mathbb{R}^{d}\right)$, and $w$ is a weak solution of $\Delta w=s$ in $\mathbb{R}^{d}$. Moreover, the function s satisfies $\int_{\mathbb{R}^{d}} s d x=1,0 \leq s \leq 2 d-1$, and $s=0$ in $\mathbb{R}^{d} \backslash B_{R}$ for some $R>0$.

Proof. Since Lemma 3.1 gives existence of limits along subsequences, we need only prove uniqueness of the limiting $s$ and $w$. By Proposition 2.4 $\Delta w=s$ almost everywhere, so it is enough to show that $w$ is unique. Suppose $w, w^{\prime} \in C\left(\mathbb{R}^{d}\right)$ are distinct and that $\bar{w}_{n_{k}} \rightarrow w$ and $\bar{w}_{n_{k}^{\prime}} \rightarrow w^{\prime}$ along subsequences locally uniformly in $\mathbb{R}^{d}$ as $k \rightarrow \infty$. Since $w=w^{\prime}=-\Phi$ outside of $B_{R}$ for some $R>0$, we may assume without loss of generality that

$$
\sup _{B_{R}}\left(w-w^{\prime}\right)>0=\sup _{\partial B_{R}}\left(w-w^{\prime}\right) .
$$


According to Proposition 2.5, we may select a point $a \in \mathbb{R}^{d}$ such that $a \neq 0, w(a)>$ $w^{\prime}(a)$, both $w$ and $w^{\prime}$ are twice differentiable at $a$, and $D^{2} w(a) \leq D^{2} w^{\prime}(a)-2 \varepsilon I$ for some $\varepsilon>0$.

Define $v:=w+\Phi$ and $v^{\prime}:=w^{\prime}+\Phi$. Since $a \neq 0, \bar{v}_{n_{k}^{\prime}} \rightarrow v^{\prime}$ uniformly in a neighborhood of $a$ as $k \rightarrow \infty$ and $v^{\prime}$ is twice differentiable at $a$. Using Lemma 4.1. we may select a $u: \mathbb{Z}^{d} \rightarrow \mathbb{Z}$ such that

$$
\Delta^{1} u(x) \leq 2 d-1 \quad \text { and } \quad u(x) \geq \frac{1}{2} x^{t}\left(D^{2} v^{\prime}(a)-\varepsilon I\right) x \quad \text { for all } x \in \mathbb{Z}^{d} .
$$

Since $D^{2} v^{\prime}(a)-\varepsilon I \geq D^{2} v(a)+\varepsilon I$ it follows that

$$
u(x) \geq \frac{1}{2} x^{t}\left(D^{2} v(a)+\varepsilon I\right) x \text { for all } x \in Z^{d} .
$$

We use a shifted version of $u$ to "lower" $v_{n_{k}}$. For the $a$ chosen above, we define

$$
h_{k}:=n_{k}^{-1 / d}, \quad a_{k}:=\left\lfloor h_{k}^{-1} a\right\rceil,
$$

and

$$
u_{n_{k}}(x):= \begin{cases}u\left(x-a_{k}\right)+\left\lfloor h_{k}^{-1} D v(a)\right\rceil \cdot\left(x-a_{k}\right) & \text { if } x \in B_{h_{k}^{-1} r}\left(a_{k}\right), \\ 1+\max _{\mathbb{Z}^{d}} v_{n_{k}} & -u(0)+v_{n_{k}}\left(a_{k}\right)-1\end{cases}
$$

We claim that for small $r>0$ and large $n_{k}$, the function $\tilde{v}:=\min \left\{v_{n_{k}}, u_{n_{k}}\right\}$ contradicts the least action principle for $v_{n_{k}}$. Since

$$
\tilde{v}\left(a_{k}\right)=u_{n_{k}}\left(a_{k}\right)=v_{n_{k}}\left(a_{k}\right)-1,
$$

it is enough to show that $n \delta_{0}+\Delta^{1} \tilde{v} \leq 2 d-1$ and $\tilde{v} \geq 0$.

To show that $n \delta_{0}+\Delta^{1} \tilde{v} \leq 2 d-1$, it is enough, by Proposition 2.1, to show that $v_{n_{k}} \leq u_{n_{k}}$ in $B_{h_{k}^{-1} r}\left(a_{k}\right) \backslash B_{h_{k}^{-1} r / 2}\left(a_{k}\right)$ and $0 \notin B_{h_{k}^{-1} r / 2}\left(a_{k}\right)$ for small $r>0$ and large $n_{k}$. Since $v$ is twice differentiable at $a$, we have, for small $r>0$ and large $n_{k}$,

$$
\bar{v}_{n_{k}}(x) \leq \bar{v}_{n_{k}}(a)+D v(a) \cdot(x-a)+\frac{1}{2}(x-a)^{t} D^{2} v(a)(x-a)+\frac{\varepsilon}{8} r^{2},
$$

for all $x \in B_{r}(a)$. Undoing the scaling, we discover that

$$
v_{n_{k}}(x) \leq v_{n_{k}}\left(a_{k}\right)+h_{k}^{-1} D v(a) \cdot\left(x-a_{k}\right)+\frac{1}{2}\left(x-a_{k}\right)^{t} D^{2} v(a)\left(x-a_{k}\right)+\frac{\varepsilon}{8} h_{k}^{-2} r^{2},
$$

for all $x \in B_{h_{k}^{-1} r}\left(a_{k}\right)$. Since

$$
u(x) \geq \frac{1}{2} x^{t} D^{2} v(a) x+\frac{\varepsilon}{4} h_{k}^{-2} r^{2},
$$

for all $x \in B_{h_{k}^{-1} r} \backslash B_{h_{k}^{-1} r / 2}$, we see that

$$
u_{n_{k}}(x) \geq v_{n_{k}}(x)+\frac{\varepsilon}{8} h_{k}^{-2} r^{2}-d^{1 / 2} h_{k}^{-1} r-u(0)-1,
$$

for all $y \in B_{h_{k}^{-1} r}\left(a_{k}\right) \backslash B_{h_{k}^{-1} r / 2}\left(a_{k}\right)$. Since the error term on the right-hand side is positive for large $n_{k}$, we see that

$$
u_{n_{k}}>v_{n_{k}} \quad \text { in } B_{h_{k}^{-1} r}\left(a_{k}\right) \backslash B_{h_{k}^{-1} r / 2}\left(a_{k}\right),
$$

for small $r>0$ and large $n_{k}$. 
To show that $\tilde{v} \geq 0$, it is enough to show that $u_{n_{k}} \geq 0$ in $B_{h_{k}^{-1} r}\left(a_{k}\right)$ for small $r>0$ and large $n_{k}$. Note that since $v$ is twice differentiable at $a, \bar{v}_{n_{k}} \rightarrow v$ in $B_{r}(a)$, and $w(a)>w^{\prime}(a)$, we have

$$
\bar{v}_{n_{k}}(a)+D v(a) \cdot(x-a)+\frac{1}{2}(x-a)^{t} D^{2} v(a)(x-a) \geq \frac{v(a)}{2}>\frac{v^{\prime}(a)}{2} \geq 0,
$$

for small $r>0$, large $n_{k}$, and $x \in B_{r}(a)$. Thus, for $x \in B_{h_{k}^{-1} r}\left(a_{k}\right)$, we may compute

$$
\begin{aligned}
u_{n_{k}}(x) & =u\left(x-a_{k}\right)+\left\lfloor h_{k}^{-1} D v(a)\right\rceil \cdot\left(x-a_{k}\right)-u(0)+v_{n_{k}}\left(a_{k}\right)-1 \\
\geq & \frac{1}{2}\left(x-a_{k}\right)^{t} D^{2} v(a)\left(x-a_{k}\right)+h_{k}^{-1} D v(a) \cdot\left(x-a_{k}\right)+v_{n_{k}}\left(a_{k}\right) \\
& \quad-u(0)-1-d^{1 / 2}\left|x-a_{k}\right| \\
& \geq h_{k}^{-2} \frac{v(a)}{2}-u(0)-1-h_{k}^{-1} d^{1 / 2} \\
\geq & 0,
\end{aligned}
$$

for small $r>0$ and large $n_{k}$.

The remaining assertions about $s$ follow immediately from (3.1) and our choice of scaling.

Remark 4.3. For those readers familiar with viscosity solutions of fully nonlinear elliptic equations (see [2]), we point out that the above proof shows that the limit $\bar{w}_{n} \rightarrow w$ is the unique solution of the obstacle problem

$$
\begin{aligned}
& w:=\inf \left\{w^{\prime} \in C\left(\mathbb{R}^{d}\right) \mid w^{\prime} \geq-\Phi \text { and } \Delta w^{\prime} \leq 2 d-1 \text { in } \mathbb{R}^{d},\right. \\
& \left.\quad \text { and } G\left(D^{2} w^{\prime}+D^{2} \Phi\right) \leq 0 \text { in } \mathbb{R}^{d} \backslash\{0\}\right\},
\end{aligned}
$$

where

$$
\begin{aligned}
& G(A):=\inf \left\{s \in \mathbb{R} \mid \text { there is a } u: \mathbb{Z}^{d} \rightarrow \mathbb{Z} \text { such that for all } y \in \mathbb{Z}^{d}\right. \\
& \left.\qquad \Delta^{1} u(y) \leq 2 d-1 \text { and } u(y) \geq \frac{1}{2} y^{t}(A-s I) y\right\},
\end{aligned}
$$

and we interpret the differential inequalities in the sense of viscosity. In fact, our proof that the limit $w$ is unique is exactly a proof that $w$ is the unique solution of (4.3) with the viscosity solution terminology stripped out.

Without too much difficulty, one can show that the operator $G$ is Lipschitz and elliptic. However, we have no reason to believe that $G$ is uniformly elliptic, and thus the existing theory of fully nonlinear elliptic obstacle problems does not apply directly. Whether this theory can be re-worked for $G$ and whether the characterization 4.3 is useful remains to be seen.

\section{REFERENCES}

[1] Per Bak, Chao Tang, and Kurt Wiesenfeld, Self-organized criticality, Phys. Rev. A (3) 38 (1988), no. 1, 364-374, DOI 10.1103/PhysRevA.38.364. MR949160 (89g:58126)

[2] Luis A. Caffarelli and Xavier Cabré, Fully nonlinear elliptic equations, American Mathematical Society Colloquium Publications, vol. 43, American Mathematical Society, Providence, RI, 1995. MR1351007 (96h:35046)

[3] Michael Christ, Lectures on singular integral operators, CBMS Regional Conference Series in Mathematics, vol. 77, Published for the Conference Board of the Mathematical Sciences, Washington, DC, 1990. MR1104656 (92f:42021)

[4] Deepak Dhar, Self-organized critical state of sandpile automaton models, Phys. Rev. Lett. 64 (1990), no. 14, 1613-1616, DOI 10.1103/PhysRevLett.64.1613. MR1044086 (90m:82053) 
[5] P. Diaconis and W. Fulton, A growth model, a game, an algebra, Lagrange inversion, and characteristic classes, Rend. Sem. Mat. Univ. Politec. Torino 49 (1991), no. 1, 95-119 (1993). Commutative algebra and algebraic geometry, II (Italian) (Turin, 1990). MR1218674 (94d:60105)

[6] Lawrence C. Evans, Weak convergence methods for nonlinear partial differential equations, CBMS Regional Conference Series in Mathematics, vol. 74, Published for the Conference Board of the Mathematical Sciences, Washington, DC, 1990. MR1034481 (91a:35009)

[7] Anne Fey, Lionel Levine, and Yuval Peres, Growth rates and explosions in sandpiles, J. Stat. Phys. 138 (2010), no. 1-3, 143-159, DOI 10.1007/s10955-009-9899-6. MR2594895 (2011c:82051)

[8] David Gilbarg and Neil S. Trudinger, Elliptic partial differential equations of second order, Classics in Mathematics, Springer-Verlag, Berlin, 2001. Reprint of the 1998 edition. MR1814364 (2001k:35004)

[9] Hung-Ju Kuo and Neil S. Trudinger, Estimates for solutions of fully nonlinear discrete schemes, Trends in partial differential equations of mathematical physics, Progr. Nonlinear Differential Equations Appl., vol. 61, Birkhäuser, Basel, 2005, pp. 275-282, DOI 10.1007/37643-7317-2-20, (to appear in print). MR2129624 (2006f:35089)

[10] Gregory F. Lawler and Vlada Limic, Random walk: a modern introduction, Cambridge Studies in Advanced Mathematics, vol. 123, Cambridge University Press, Cambridge, 2010. MR2677157

[11] Lionel Levine and Yuval Peres, Strong spherical asymptotics for rotor-router aggregation and the divisible sandpile, Potential Anal. 30 (2009), no. 1, 1-27, DOI 10.1007/s11118-008-9104-6. MR2465710 (2010d:60112)

[12] Lionel Levine and James Propp, What is ... a sandpile?, Notices Amer. Math. Soc. 57 (2010), no. 8, 976-979. MR2667495

[13] Srdjan Ostojic, Patterns formed by addition of grains to only one site of an abelian sandpile, Physica A 318 (2003), no. 1-2, 187-199, DOI 10.1016/S0378-4371(02)01426-7.

Courant Institute at New York University, 251 Mercer Street, New York, NY 10012

E-mail address: pegden@math.nyu.edu

Courant Institute at New York University, 251 Mercer Street, New York, NY 10012

E-mail address: csmart@math.nyu.edu 